\title{
Natural History of an Untreated Type 1 Endoleak: A Case Report
}

\author{
Alice Shen ${ }^{1}$, Gabriel O. Ologun ${ }^{1}$, Hilary Keller ${ }^{1}$, Lawrence Sampson ${ }^{2}$ \\ 1. General Surgery, Guthrie Clinic/Robert Packer Hospital 2. Vascular Surgery, Guthrie Clinic/Robert \\ Packer Hospital
}

$\square$ Corresponding author: Gabriel O. Ologun, gabe.ologun@gmail.com

Disclosures can be found in Additional Information at the end of the article

\section{Abstract}

We present a case of giant abdominal aortic aneurysm greater than $17 \mathrm{~cm}$ complicated by an endoleak, demonstrating the natural history of an untreated Type 1 endoleak.

Categories: Cardiac/Thoracic/Vascular Surgery, Medical Education, Family/General Practice Keywords: endovascular aneurysm repair (evar), endoleak, abdominal aortic aneurysm (aaa)

\section{Introduction}

Abdominal aortic aneurysms are the most common type of aortic aneurysm seen in $3 \%$ - $9 \%$ of patients ages greater than 50 years in the Western world. Ruptured abdominal aortic aneurysms are the 10th leading cause of death in men above 55 years old [1]. The advancement of endovascular aneurysm repair has lowered the perioperative mortality compared to open repair. We present a case of a giant abdominal aortic aneurysm after endovascular repair. Informed consent was obtained for this case report, images, and publication.

\section{Case Presentation}

The patient is a 67-year-old male with a history of hypertension, chronic obstructive pulmonary disease, and a known infrarenal abdominal aortic aneurysm (AAA) who presented to the emergency department with abdominal pain of about six to eight hours duration. He was on antihypertensive, statin, and antiplatelet medications. He developed sudden sharp pains in his mid to lower abdomen. He had moderate pain. He denied nausea, vomiting, diarrhea, melena, or hematochezia.

Received 06/13/2017 Review began 06/28/2017 Review ended 07/13/2017 Published 07/24/2017

C) Copyright 2017

Shen et al. This is an open access article distributed under the terms of the Creative Commons Attribution License CC-BY 3.0., which permits unrestricted use, distribution, and reproduction in any medium, provided the original author and source are credited.
Of note, the patient had an open AAA repair with a graft for rupture with subsequent AAA growth secondary to endotension, resulting in an endovascular revision and stent placement at an outside facility. He had been followed in our institution's vascular department for over a year for surveillance per request from his primary physician due to the gradual growth in the size of his AAA. Treatment options were discussed, including open versus endovascular approaches, plus a second opinion was obtained from another vascular surgeon in our facility. The patient declined any surgical or endovascular intervention, accepting his risk of mortality from sudden rupture.

This patient's vital signs were within normal ranges, except for a sinus tachycardia of 104 and systolic blood pressure ranging from 90 - 110. On examination, the patient was uncomfortable. He had biphasic Doppler signals in his distal lower extremities bilaterally. The abdomen was softly distended and mildly tender with an appreciable pulsatile mass. 


\section{Cureus}

A computed tomographic angiogram (CT angio) demonstrated an AAA with evidence of interval increase in size from $16.3 \mathrm{~cm}$ to $17.3 \mathrm{~cm}$ within the past six months with evidence of a Type 1 endoleak identified near the central portion of the aortic graft (Figure 1). At this point, the vascular surgery team was consulted to evaluate the patient.
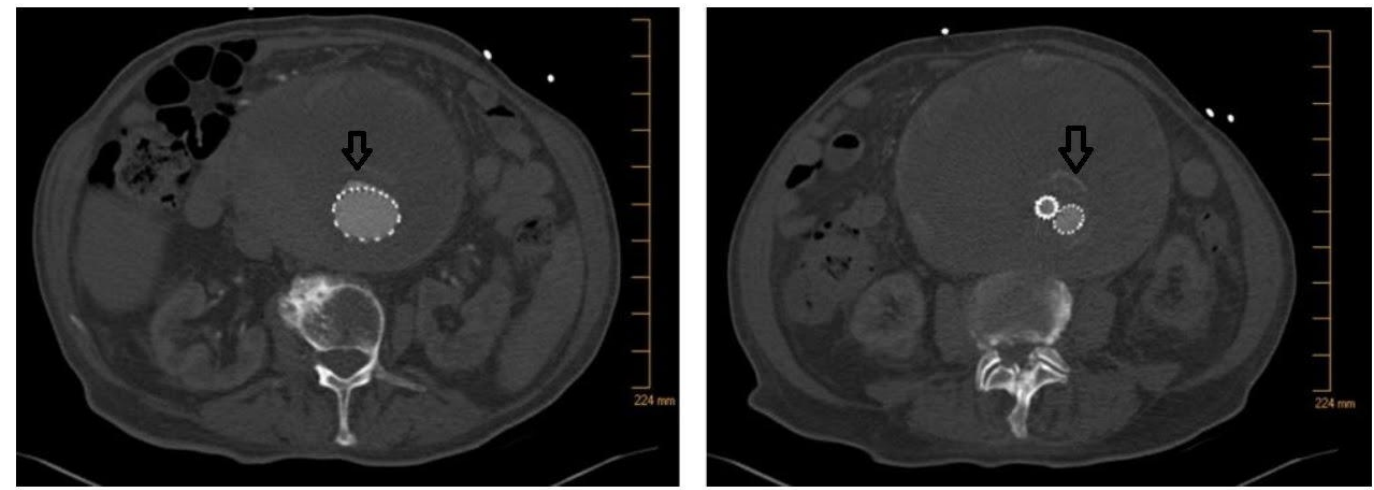

\section{FIGURE 1: Axial computed tomographic angiography of an abdominal aortic aneurysm greater than $17 \mathrm{~cm}$ with arrow showing the endoleak}

Computed tomographic angiography (CT angio) results were discussed with the patient and his family. The patient again declined against any surgical intervention. He opted for palliative/comfort care measures. Shortly thereafter, the patient became hypotensive, unconscious, and pulseless, and he was pronounced dead at that time. The family declined an autopsy.

\section{Discussion}

This is an interesting case presentation of the natural history of an untreated Type 1 endoleak noted on CT angio. AAA is the most common type of aortic aneurysm and is seen in $3 \%$ to $9 \%$ of patients over the age of 50 years in the Western world [1]. Since the introduction and rapid advancement of endovascular aneurysm repair (EVAR) in the 1990s, multiple prospective randomized trials have shown that the endovascular technique lowers the 30-day perioperative mortality in contrast to open repair (1.7\% versus $4.7 \%$, respectively); the all-cause mortality was not different [2-5]. However, EVAR has a higher long-term complication rate than open AAA repair, and endoleaks are unique complications. Endoleaks are defined as persistent blood flow in an aneurysm outside the prosthesis, causing enlargement of the aneurysm sac. They have been classified into five types according to etiology. They are summarized as the following:

Type I: Leakage from the proximal or distal anchoring points from an ineffective seal Type II: Leakage from the retrograde flow through the lateral branches (e.g., lumbar artery or inferior mesenteric artery) not covered by the graft. This is the most common type. Type III: Leakage from the overlap zones of the individual stent prosthesis components Type IV: Leakage through the stent material, now rare as a result of improved stent prosthesis Type V: "Endotension" seen on follow-up imaging as an increase in aneurysm size without contrast extravasation outside of the stent prosthesis itself. This is thought to be caused by increased pressure.

Patients who have had EVAR require lifelong surveillance because endoleaks can lead to 
aneurysm expansion and rupture. Each endoleak type has its own algorithm of intervention directed at treating the underlying etiology. Type I and III endoleaks represent direct communication with the systemic blood flow and require immediate repair. They can be approached endovascularly or via conversion to an open procedure. Type II endoleaks can spontaneously thrombose and immediate intervention is not needed in an asymptomatic patient. Type IV endoleaks require stent replacement, but this has been rare due to the improved prosthetic material. Type $\mathrm{V}$ endoleaks often require conversion to open repair, but continued nonoperative management is also acceptable [6].

\section{Conclusions}

This case presents a patient with a giant AAA secondary to a Type I endoleak after a prior open repair who declined any surgical intervention and ultimately succumbed to his disease. Despite the large aneurysm size and continued expansion, this patient was asymptomatic and survived more than seven years after the endovascular revision of his previous open AAA repair. Moreover, this patient survived 17 years after the initial ruptured native AAA that required open repair. The risk of revisional surgery versus the risk of rupture with watchful waiting in elderly patients remains an ongoing debate.

\section{Additional Information}

\section{Disclosures}

Human subjects: Consent was obtained by all participants in this study. Conflicts of interest: In compliance with the ICMJE uniform disclosure form, all authors declare the following: Payment/services info: All authors have declared that no financial support was received from any organization for the submitted work. Financial relationships: All authors have declared that they have no financial relationships at present or within the previous three years with any organizations that might have an interest in the submitted work. Other relationships: All authors have declared that there are no other relationships or activities that could appear to have influenced the submitted work.

\section{Acknowledgements}

We would like to thank Danielle A. Pigneri and Andrew Trecartin for proofreading and editing the manuscript.

\section{References}

1. Wilmink $A B$, Quick CR: Epidemiology and potential for prevention of abdominal aortic aneurysm. Br J Surg. 1998, 85:155-62.

2. Becquemin JP: The ACE trial: a randomized comparison of open versus endovascular repair in good risk patients with abdominal aortic aneurysm. J Vasc Surg. 2009, 50:222-24. 10.1016/j.jvs.2009.04.074

3. Lederle FA, Freischlag JA, Kyriakides TC, et al.: Outcomes following endovascular vs open repair of abdominal aortic aneurysm: a randomized trial. JAMA. 2009, 302:1535-42. 10.1001/jama.2009.1426

4. EVAR trial participants: Endovascular aneurysm repair versus open repair in patients with abdominal aortic aneurysm (EVAR trial 1): randomised controlled trial. Lancet. 2005, 365:2179-86. 10.1016/S0140-6736(05)66627-5

5. Prinssen M, Buskens E, Blankensteijn JD: The Dutch Randomised Endovascular Aneurysm Management (DREAM) trial. Background, design and methods. J Cardiovasc Surg (Torino). 2002, 43:379-84.

6. White SB, Stavropoulos SW: Management of endoleaks following endovascular aneurysm repair. Semin Intervent Radiol. 2009, 26:33-38. 10.1055/s-0029-1208381 